\title{
NASA Glenn Research Center Solar Cell Experiment onboard the International Space Station
}

\author{
Matthew G. Myers ${ }^{1}$, David S. Wolford, Norman F. Prokop ${ }^{1}$, Michael J. Krasowski ${ }^{1}$, David S. Parker ${ }^{2}$, Justin \\ C. Cassidy ${ }^{2}$, William E. Davies ${ }^{3}$, Janelle O. Vorreiter ${ }^{4}$, Michael F. Piszczor ${ }^{1}$, Jeremiah S. Mcnatt ${ }^{1}$, Danny C. \\ Spina $^{1}$ \\ ${ }^{1}$ NASA Glenn Research Center, Cleveland, Ohio 44135 \\ ${ }^{2}$ Goddard Space Flight Center, Code 408/ Lockheed Martin, Greenbelt MD 20771 \\ ${ }^{3}$ Goddard Space Flight Center, Code 408 /Jackson and Tull, Greenbelt MD 20771 \\ ${ }^{4}$ Goddard Space Flight Center, Code 408 / ATK Space Systems, Greenbelt MD 20771
}

\begin{abstract}
Accurate air mass zero (AM0) measurement is essential for the evaluation of new photovoltaic (PV) technology for space solar cells. The NASA Glenn Research Center (GRC) has flown an experiment designed to measure the electrical performance of several solar cells onboard NASA Goddard Space Flight Center's (GSFC) Robotic Refueling Mission's (RRM) Task Board 4 (TB4) on the exterior of the International Space Station (ISS). Four industry and government partners provided advanced PV devices for measurement and orbital environment testing. The experiment was positioned on the exterior of the station for approximately eight months, and was completely selfcontained, providing its own power and internal data storage. Several new cell technologies including four-junction (4J) Inverted Metamorphic Multi-junction (IMM) cells were evaluated and the results will be compared to ground-based measurement methods.
\end{abstract}

Index Terms - air mass zero, international space station, low earth orbit environment, photovoltaic cells, solar cell calibration.

\section{INTRODUCTION}

NASA and other aerospace entities use a variety of methods to measure the performance of space solar cells. The most common method of measuring air mass zero (AM0) performance is the laboratory solar simulator. Once calibrated with reference standards, the solar simulator can measure large numbers of cells in order to predict in-space cell performance. Obtaining directly measured AM0 primary reference standards is an on-going challenge for the space PV community.

In 2012, center leadership at GSFC and GRC began a dialogue to identify a suitable collaborative objective that would grant access to space via the RRM project. The RRM is a multi-mission program to demonstrate tools and techniques for robotic satellite servicing. It utilizes the Canadian Space Agency's (CSA) Special Purpose Dexterous Manipulator (SPDM), also known as Dextre, onboard the ISS. The highly versatile SPDM is operated at the end of CSA's Canadarm 2 (SSRMS). Now in its second phase, RRM is

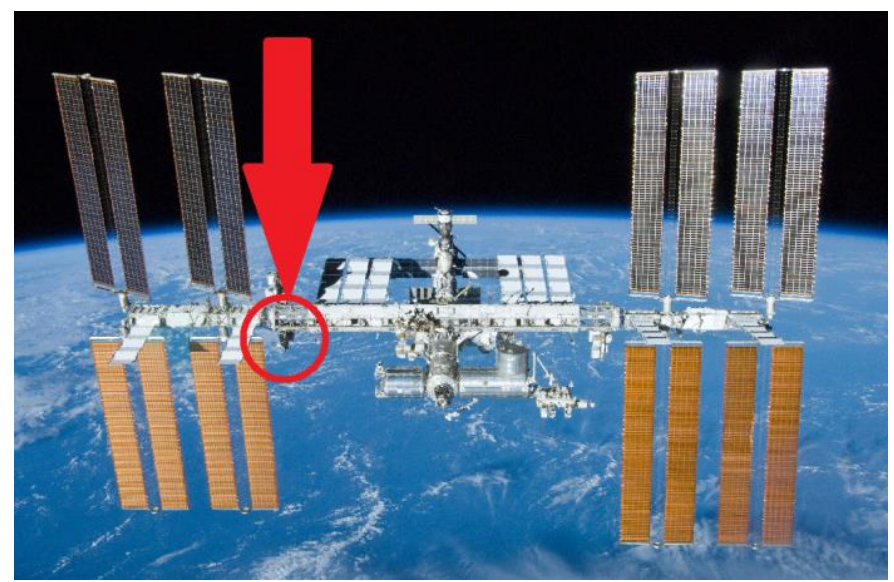

Fig. 1. Image showing the position of the SCE on the ISS when mounted externally.

managed by GSFC's Satellite Servicing Capabilities Office (SSCO).

An experiment was developed and integrated into TB4, a removable sub-module of the RRM, which includes both electrical measurement and exposure testing of advanced PV devices. Electrical measurement is performed when the task board is being deployed and recovered by the robotic arm. During this operation, the arm directs the cells on the active side of the solar cell experiment (SCE) at the sun while avoiding unwanted reflected light. Cells and materials on the passive side of the SCE are included for full orbital environment exposure. The location of TB4 on the ISS is shown in figure 1.

The cells and materials being evaluated were provided by several industry and government partners including MicroLink Devices, Sandia National Laboratories, Solaero Technologies, and Spectrolab. These devices were evaluated before flight in the solar cell measurement labs at GRC, and will be reevaluated following their return to earth. The Mobile and Remote Sensing Laboratory (MARS Lab) team at GRC was tasked with the development of the data acquisition system for 
the active side of the experiment, as team members had participated in previous space PV measurement experiments including MISSE 5, 7 and 8 [1]-[3]. The team designed, built, and delivered the flight hardware on an extremely accelerated timeline during December of 2013 and January of 2014. The integration of the experiment into TB4 as well as preflight qualification tests were conducted at GSFC between February 7 and April 12, 2014. Afterward, TB4 was shipped to Johnson Space Center for final launch preparations.

TB4 was launched on the European Space Agency's Automated Transfer Vehicle "Georges Lemaitre" (ATV-5) on July 29th 2014 from the European Spaceport in French Guiana. It remained inside the ISS for around nine months before it was deployed via the Japanese Experiment Module (JEM/Kibo) air-lock and slide table.

\section{EXPERIMENT DESCRIPTION}

The SCE consists of two parts located on opposite sides of TB4, a sub module of the RRM about the size of a large briefcase. The passive area, mounted on the front of TB4, is exposed to the LEO environment for the duration of the flight facing the ram direction. These cells are exposed to widely varying solar illumination, atomic oxygen, micrometeoroids and other LEO environmental factors. The passive experiment samples are not instrumented for measurement during flight. The active area, mounted on the back side of TB4, is normally shielded from the sun and other LEO environmental factors by the RRM structure to which TB4 is mounted. The active part of the experiment is designed to power up and record data when aimed at the sun by the SPDM. The data acquisition system for cell measurement is contained inside of TB4. All devices under test are mounted to aluminum plates covered with Kapton tape and a $\mathrm{SiO}_{2}$ coating. Cells are bonded directly to the $\mathrm{SiO}_{2}$ coated Kapton with CV10-2568 silicone adhesive. Interconnects are soldered to twisted pair 26 gauge wires. One twisted pair is connected to each electrical lead as is required in order to make a four-wire measurement. A conformal coating is used on soldered electrical connections and wherever the $\mathrm{SiO}_{2}$ coating is compromised. Wires are staked to the Kapton to direct wires to feed through slots.

The experiment is entirely self-contained, with power, instrument control, and data storage being housed within TB4. There are no electrical interfaces with ISS systems. This allowed the experiment to be developed and flown in a very short time, however because of this design, the success of the active side of the experiment will be impossible to determine until the experiment is recovered.

\section{A. Active Experiment}

The active measurement experiment consists of 5 plates. A large plate contains a string of four Solero Technologies conventional ZTJ cells and are the source of power for the experiment. Two medium sized plates are populated by Solaero Technologies next generation IMM 4J solar cells, with one plate holding a string of 3 cells and the other plate holding three separately measured cells. On a fourth plate, Spectrolab provided two GaInP component sub-cells, with one from a current generation XTJ triple junction cell and the other from a next generation 4J IMM cell. On the last plate, MicroLink Devices supplied two next generation 3J IMM cells. Figure 2 shows the cells for active measurement on the back of TB4.

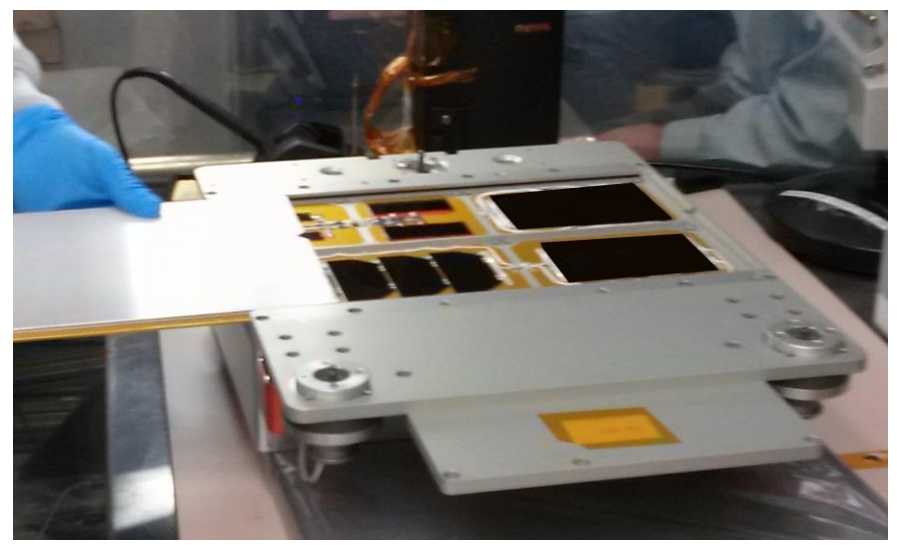

Fig. 2. Sample cells located on the back side of TB4. The protective cover is being removed. Detail has been obscured (GRC).

\section{B. Passive Experiment}

The passive experiment consists of 2 plates. The larger plate is holds four MicroLink 3J IMM cells. The smaller plate has two microarray devices provided by Sandia National Laboratories. The passive experiment cells are configured with a ballast resistor to simulate a load. The Air Force Research Laboratory contributed three adhesive test samples to the smaller plate as well. These samples will tested for changes in the optical absorption of the adhesive due to the LEO environment. The flight hardware for the passive portion of SCE is shown in figure 3 


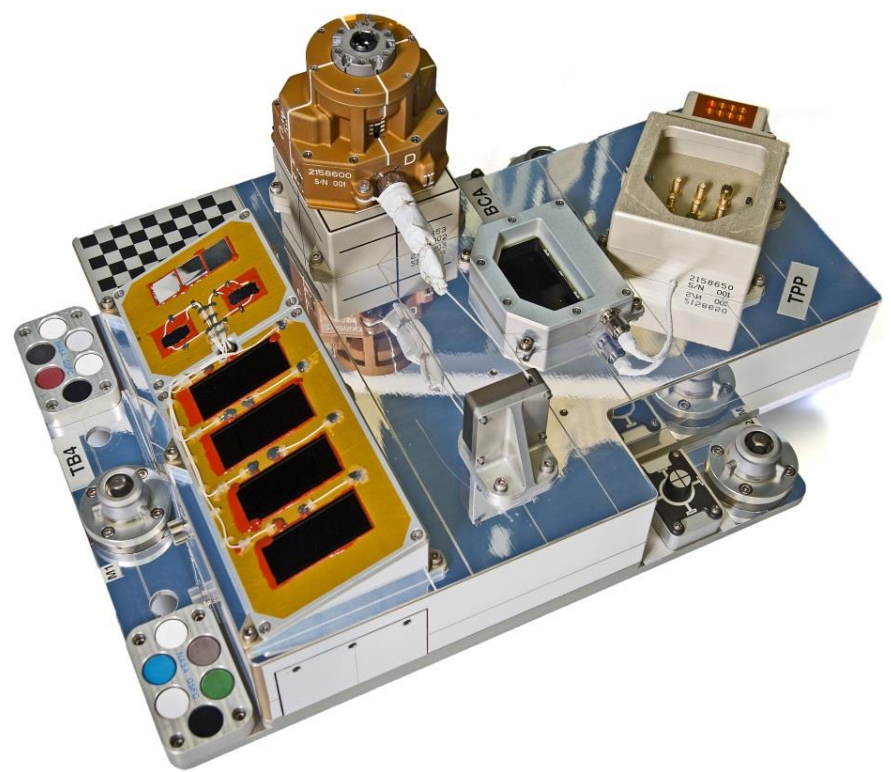

Fig. 3. Passive experiment samples on the front side of TB4.

\section{Electrical Measurement System}

The measurement electronics were designed and fabricated by the MARS lab at GRC. They consist of two printed wiring assemblies (PWA) which can each measure four solar cell circuits and their corresponding temperature sensors. The processor becomes active when sufficient power is being generated by the four cell power string. Power must be maintained for one minute before data is collected. The one minute delay is to minimize false starts and unproductive use of flash memory. Each board sequentially scans through its four channels. IV curves are taken of each channel and are preceded and followed by a temperature reading from an AD590LF integrated circuit temperature sensor located on the back of the plate near the center of the corresponding cell or string. Scans are performed at one minute intervals while solar illumination is sufficient. The boards safely power down when the input voltage is less than seven volts.

Each PWA has the memory capacity to save 2044 sets of four channel scans each. The one minute interval between data sets allows for a minimum of 34 hours of continuous data collection. When the flash memory is at capacity, the instrument is designed to stop recording and avoid overwriting existing data.

\section{Mission Procedure}

The deployment of the SCE took place on May 2nd 2015. TB4 was removed from the JEM airlock using the SSRMS with attached SPDM. The robotic arm was then used to aim the active side of the SCE directly at the sun for the daytime segment of one orbit. Sun alignment was accomplished using the tool alignment camera. This camera, located on the SPDM, was oriented normal to the face of the cells under test, and had a crosshair overlay that allowed for precise aiming of the experiment. Figures 4 and 5 show TB4 being directed at the sun by the SPDM and the view from the camera respectively. The sun appears as a dark spot in the center of the camera view due to sensor saturation.

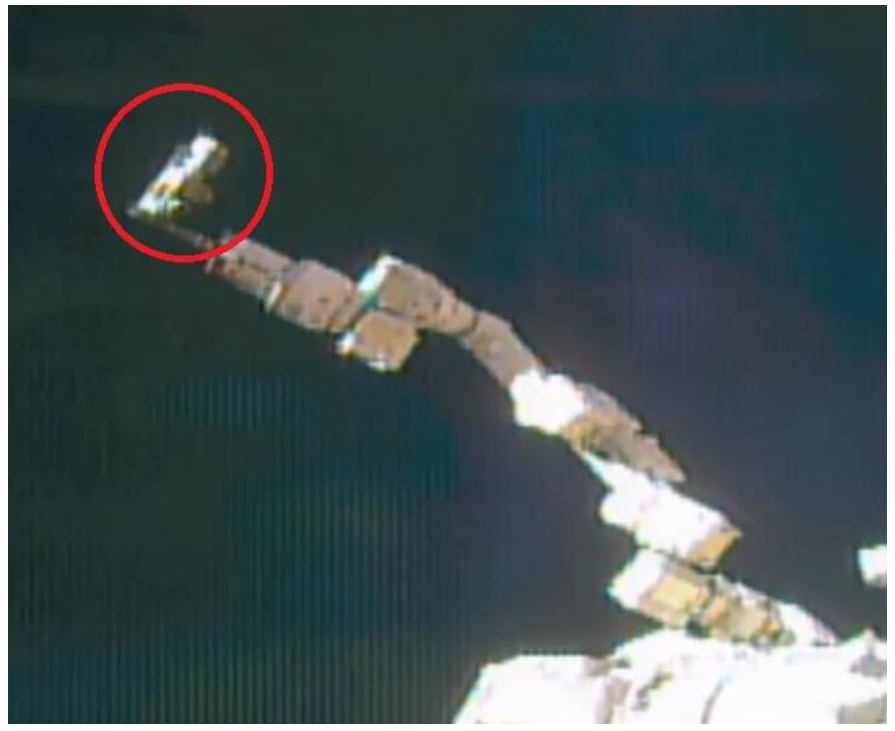

Fig. 4. TB4 (circled in red) being directed at the sun.

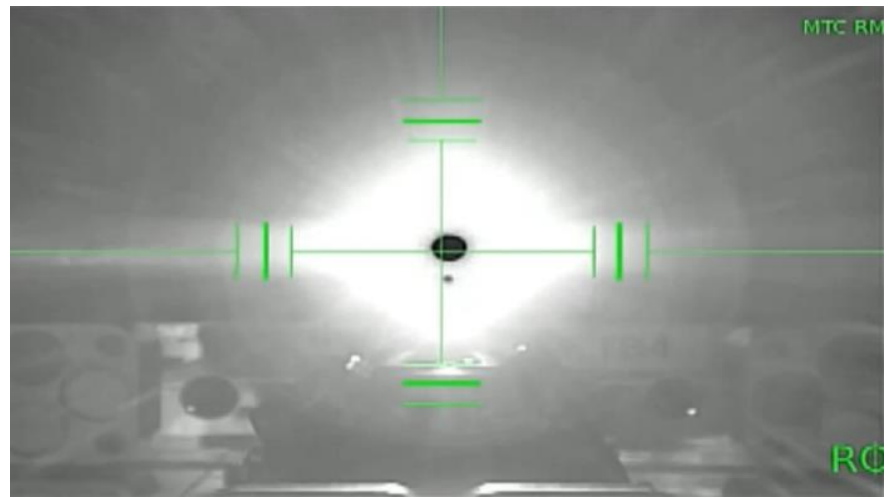

Fig. 5. Image from tool camera used for sun alignment.

While the cells were illuminated, the measurement electronics were powered up automatically by the ZTJ array. IV curves with 128 points were collected along with device temperatures once every minute. This cycle continued until sun tracking was stopped and the measurement electronics powered down.

After this initial exposure, the SPDM was used to bolt TB4 to the RRM structure with the active side down. Over the next eight months, the cells on the passive side of the experiment were exposed to the LEO environment facing the ram direction.

On January $6^{\text {th }}, 2016$, the second exposure took place. The SPDM was first used to unbolt TB4 from its place on the RRM. The arm then directed both sides of TB4 towards an ISS external camera to inspect for any damage the cells may have incurred during their time in the LEO environment. 
Figure 6 shows an image of the active side of the experiment during this inspection. The active side of the experiment was then again directed towards the sun. This time data was collected for the daytime segments of two full orbits. Following the exposure, the SPDM delivered TB4 to the slide table of the JEM airlock for recovery to the inside of the ISS.

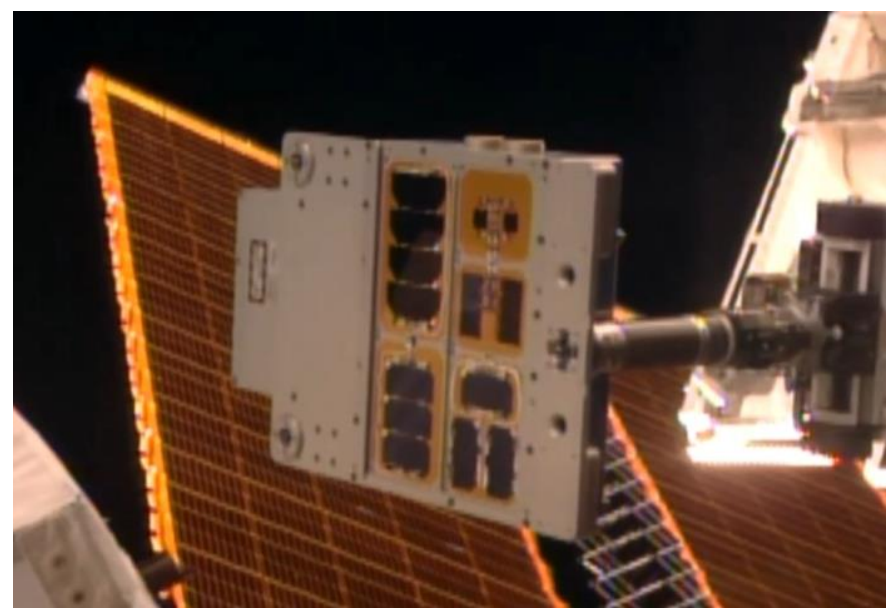

Figure 6. Image of the active side of the experiment during inspection

The experiment returned to earth on SpaceX CRS-8, which splashed down May $11^{\text {th }}$ of 2016. At the time of this papers submission, TB4 was making its way to GSFC where the data from the flash memory of the data acquisition system will be recovered and evaluated. The plates containing the cells will be returned to GRC where post-flight testing and evaluation will be conducted. Results from the experiment were unfortunately unavailable by the submission date of this paper, however will be reported in the near future.

\section{SUMMARY}

In collaboration with GSFC, GRC has flown a solar cell characterization experiment on the ISS. The SCE takes advantage of access to space via SSCO's RRM TB4. Ten advanced AM0 solar cells from three industry partners, have had their electrical characteristics measured in space. An additional nine samples, from three industry and government partners, were exposed for eight months to the LEO environment. The data from this experiment should provide valuable performance metrics of emerging space PV technology to manufacturers. NASA hopes to benefit from the opportunity to verify its laboratory measurement accuracy and monitor PV technological progress. This type of effort is important for continuing the mutually beneficial collaborative relationship NASA has with the space PV community.

\section{ACKNOWLEDGEMENT}

The authors would like to thank and recognize the RRM team at Goddard Space Flight Center for all their hard work in making this experiment possible, Lawrence Greer, George Baaklini, and the rest of the Glenn Research Center MARS lab for their tireless efforts in developing the measurement electronics under an extremely tight schedule, and the ROBO team at Johnson Space Center for their steady aim tracking the sun during exposure. Our industry and government partners including MicroLink Devices, Sandia National Laboratories, Solaero Technologies, and Spectrolab also have our deep appreciation for supporting this effort by supplying and preparing the test articles for the experiment.

\section{REFERENCES}

[1] M. J. Krasowski, L. C. Greer, J. M. Flatico, P. P. Jenkins and D. C. Spina, "A Hardware and Software Perspective of the Fifth Materials on the International Space Station Experiment (MISSE-5)," NASA/TM-2005-213840, 2005.

[2] Jenkins, P., Walters, R., Krasowski, M., Chapman, J., Ballard, P., Vasquez, J., Mahony, D., LaCava, S., Braun, W., Prokop, N., Flatico, J., Greer, L., Gibson, K., Kinard, W., Pippin, H., "MISSE-7: Building a Permanent Environmental Testbed for the International Space Station," Proceedings of the 9th International Space Conference Protection of Materials and Structures From Space Environment, (ICPMSE-9) MISSE Symposium, Toronto, Canada, 19-23 May 2008, AIP Conference Proceedings, Vol. 1087, p. 273, 2009.

[3] R.J. Walters et al., "Materials on the International Space Station Forward Technology Experiment" NASA/CP 2005-2134 